\title{
Low-Mach-number turbulence in interstellar gas revealed by radio polarization gradients
}

\author{
B. M. Gaensler ${ }^{1}$, M. Haverkorn ${ }^{2,3,4}$, B. Burkhart ${ }^{5}$, K. J. Newton-McGee ${ }^{1,6}$, R. D. Ekers ${ }^{6}$, A. Lazarian ${ }^{5}$, N. M. McClure-Griffiths ${ }^{6}$, \\ T. Robishaw ${ }^{1}$, J. M. Dickey ${ }^{7} \&$ A. J. Green ${ }^{1}$
}

The interstellar medium of the Milky Way is multiphase ${ }^{1}$, magnetized $^{2}$ and turbulent ${ }^{3}$. Turbulence in the interstellar medium produces a global cascade of random gas motions, spanning scales ranging from 100 parsecs to 1,000 kilometres (ref. 4). Fundamental parameters of interstellar turbulence such as the sonic Mach number (the speed of sound) have been difficult to determine, because observations have lacked the sensitivity and resolution to image the small-scale structure associated with turbulent motion ${ }^{5-7}$. Observations of linear polarization and Faraday rotation in radio emission from the Milky Way have identified unusual polarized structures that often have no counterparts in the total radiation intensity or at other wavelengths ${ }^{8-12}$, and whose physical significance has been unclear ${ }^{13-15}$. Here we report that the gradient of the Stokes vector $(Q, U)$, where $Q$ and $U$ are parameters describing the polarization state of radiation, provides an image of magnetized turbulence in diffuse, ionized gas, manifested as a complex filamentary web of discontinuities in gas density and magnetic field. Through comparison with simulations, we demonstrate that turbulence in the warm, ionized medium has a relatively low sonic
Mach number, $M_{\mathrm{s}} \lesssim 2$. The development of statistical tools for the analysis of polarization gradients will allow accurate determinations of the Mach number, Reynolds number and magnetic field strength in interstellar turbulence over a wide range of conditions.

We consider radio-continuum images of an $18-\mathrm{deg}^{2}$ patch $^{11,16}$ of the Galactic plane, observed with the Australia Telescope Compact Array (ATCA) at a frequency of $1.4 \mathrm{GHz}$. Data were simultaneously recorded in total intensity (Stokes parameter $I$ ) and in linear polarization (Stokes parameters $Q$ and $U$ ). The Stokes $I$ image (Fig. 1) shows a typical distribution of radio emission, consisting of supernova-remnant shells, ionized regions around massive stars ( $\mathrm{H}$ II regions) and unresolved distant radio sources. However, the corresponding images of $Q, U$ and the linearly polarized intensity $P \equiv\left(Q^{2}+U^{2}\right)^{1 / 2}$ in Fig. 1 are filled with complex structure that bears little resemblance to the Stokes $I$ image, as has also been seen in many other polarimetric observations at radio frequencies ${ }^{8,9,12}$. The intensity variations seen in $Q, U$ and $P$ are the result of small-scale angular structure in the Faraday rotation induced by ionized gas ${ }^{8}$, and are thus an indirect representation of

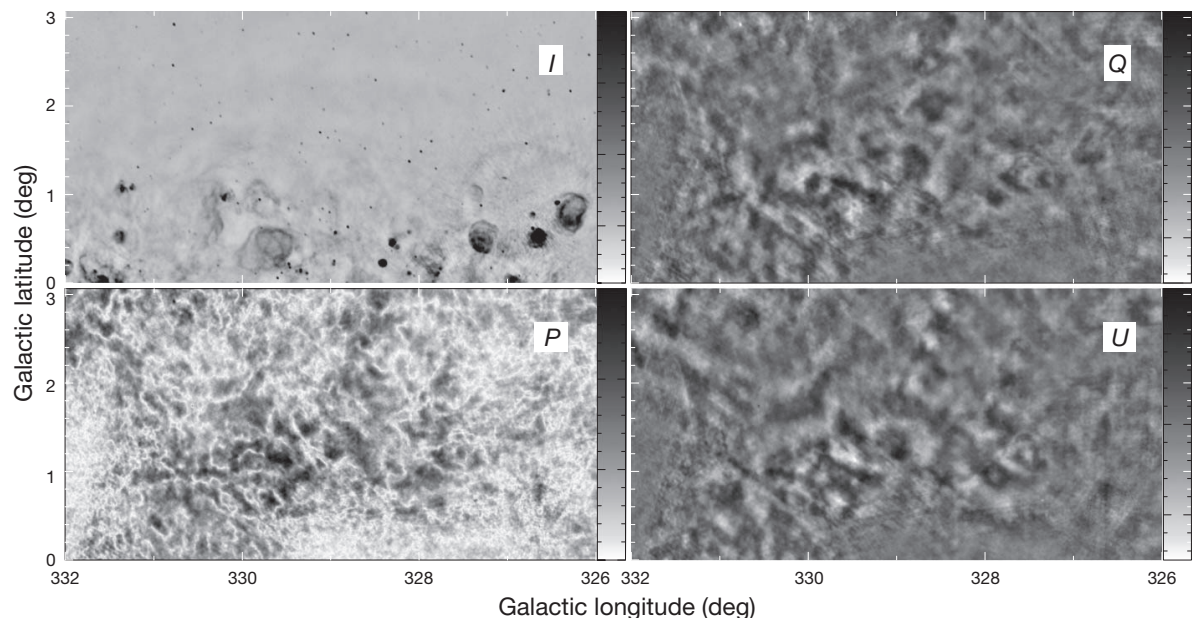

Figure $1 \mid$ Total intensity $(I)$ and linearly polarized intensity $(Q, U, P)$ for an 18-deg ${ }^{2}$ region of the Southern Galactic Plane Survey ${ }^{29}$. All four images were generated ${ }^{11}$ from a set of observations ${ }^{16}$ taken at the ATCA over the period 1997 April to 1998 April using a 96-MHz bandwidth centred on an observing frequency of $1,384 \mathrm{MHz}$. The field is a mosaic of 190 pointings each with a total integration time of $20 \mathrm{~min}$, resulting in an approximately uniform sensitivity, over most of the field, of $0.8 \mathrm{mJy}$ per beam (Stokes $I$ ) or $0.55 \mathrm{mJy}$ per beam (Stokes $Q$ and $U)$ at an angular resolution of $75 \operatorname{arcsec}\left(1 \mathrm{Jy} \equiv 10^{-26} \mathrm{~W} \mathrm{~m}^{-2} \mathrm{~Hz}^{-1}\right)$. The scale for each image is shown on the right of each panel. The Stokes $I$ image is displayed over a range of -40 to $+150 \mathrm{mJy}$ per beam (each interval corresponds to $10 \mathrm{mJy}$ per beam). Because the ATCA is an interferometer, it is not sensitive to structure on angular scales larger than $35 \mathrm{arcmin}$. Faint wisps can be seen, corresponding to the sharp edges of large-scale structures.

However, the bulk of the smooth radio emission from Galactic cosmic rays is not detected. Imaging artefacts in the form of grating rings and radial streaks can be seen around a few very bright sources, but these regions were not used in our statistical analysis. The Stokes $Q$ and $U$ images are displayed over a range of -15 to $+15 \mathrm{mJy}$ per beam (interval, $2 \mathrm{mJy}$ per beam), and the $P$ image covers a range of 0 to $15 \mathrm{mJy}$ per beam (interval, $1 \mathrm{mJy}$ per beam). Almost none of the structure seen in $Q, U$ and $P$ has any correspondence with any emission seen in Stokes I; the mottled structure results from spatial fluctuations in Faraday rotation in the ISM. 
turbulent fluctuations in the free-electron density and magnetic field throughout the interstellar medium ${ }^{15}$ (ISM).

A limitation of previous studies is that they usually interpreted the data in terms of the amplitude, $P$, and/or the angle, $\theta \equiv(1 / 2) \tan ^{-1}(U / Q)$, of the complex Stokes vector $\mathbf{P} \equiv(Q, U)$. However, neither polarization amplitude nor polarization angle is preserved under arbitrary translations and rotations in the $Q-U$ plane. These can result from one or more of a smooth distribution of intervening polarized emission, a uniform screen of foreground Faraday rotation, and the effects of missing large-scale structure in an interferometric data set. In the most general case, we are thus forced to conclude that the observed values of $P$ and $\theta$ do not have any physical significance, and that only measurements of quantities that are both translationally and rotationally invariant in the $Q-U$ plane can provide insight into the physical conditions that produce the observed polarization distribution.

The simplest such quantity is the spatial gradient of $\mathbf{P}$, that is, the rate at which the polarization vector traces out a trajectory in the $Q-U$ plane as a function of position on the sky. The magnitude of the gradient is unaffected by rotation and translation, and so has the potential to reveal properties of the polarization distribution that might otherwise be hidden by excess foreground emission or Faraday rotation, or in data sets from which large-scale structure is missing (as is the case for the data shown in Fig. 1). The magnitude of the polarization gradient is

$$
|\nabla \mathbf{P}|=\sqrt{\left(\frac{\partial Q}{\partial x}\right)^{2}+\left(\frac{\partial U}{\partial x}\right)^{2}+\left(\frac{\partial Q}{\partial y}\right)^{2}+\left(\frac{\partial U}{\partial y}\right)^{2}}
$$

The expression in equation (1) can be calculated simply, and the corresponding image of $|\nabla \mathbf{P}|$ (Fig. 2) reveals a complex network of tangled filaments. In particular, all regions in which $|\nabla \mathbf{P}|$ is high consist of elongated, narrow structures rather than extended patches. In the inset of Fig. 2, we plot the direction of $\nabla \mathbf{P}$ for a small subregion of the image, demonstrating that $\nabla \mathbf{P}$ changes most rapidly along directions oriented perpendicular to the filaments. We can explore the

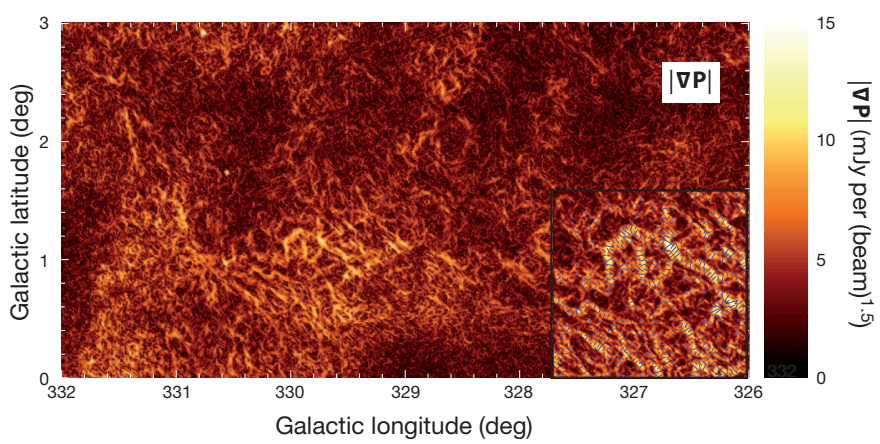

Figure $2|\nabla \mathrm{P}|$ for an 18- $\mathrm{deg}^{2}$ region of the Southern Galactic Plane Survey. $|\nabla \mathbf{P}|$ has been derived by applying equation (1) to the $Q$ and $U$ images from Fig. 1; note that $|\nabla \mathbf{P}|$ cannot be constructed from the scalar quantity $P \equiv\left(Q^{2}+U^{2}\right)^{1 / 2}$, but is derived from the vector field $\mathbf{P} \equiv(Q, U) .|\nabla \mathbf{P}|$ is a gradient in one dimension, for which the appropriate units are (beam) $)^{-0.5}$. Because $P$ measures linearly polarized intensity in units of millijanskys per beam, $|\nabla \mathbf{P}|$ has units of millijanskys per (beam) ${ }^{1.5}$. The scale showing $|\nabla \mathbf{P}|$ is shown on the right of the image, and ranges from 0 to $15 \mathrm{mJy}$ per (beam $)^{1.5}$. The inset shows an expanded version of the structure with highest $|\nabla \mathbf{P}|$, covering a box of side 0.9 deg centred on Galactic longitude $329.8 \mathrm{deg}$ and Galactic latitude $+1.0 \mathrm{deg}$. Plotted in the inset is the direction of $\nabla \mathbf{P}$ at each position, defined as $\arg (\nabla \mathbf{P}) \equiv$

$\tan ^{-1}\left[\operatorname{sign}\left(\frac{\partial Q}{\partial x} \frac{\partial Q}{\partial y}+\frac{\partial U}{\partial x} \frac{\partial U}{\partial y}\right) \sqrt{\left(\frac{\partial Q}{\partial y}\right)^{2}+\left(\frac{\partial U}{\partial y}\right)^{2}} / \sqrt{\left(\frac{\partial Q}{\partial x}\right)^{2}+\left(\frac{\partial U}{\partial x}\right)^{2}}\right]$

For clarity, vectors are shown only at points where the amplitude of the gradient is greater than $5 \mathrm{mJy}$ per (beam) ${ }^{1.5}$. frequency dependence of these filaments, because the $1.4-\mathrm{GHz}$ ATCA data shown in Fig. 1 consist of nine independent spectral channels of width $8 \mathrm{MHz}$, spread over a total bandwidth of $96 \mathrm{MHz}$. We have constructed images of $|\nabla \mathbf{P}|$ for each individual spectral channel, and these show the same set of specific features as in the overall image, albeit at reduced signal-to-noise ratios. The lack of frequency dependence indicates that the high-gradient structures seen in this data set correspond to physical features in the ISM rather than to contour lines introduced by the particular combination of observing frequency and angular resolution used ${ }^{15,17}$

We first consider the possibility that these filaments of high gradient are intrinsic to the source of emission. Abrupt spatial transitions in the strength or geometry of the magnetic field in a synchrotron-emitting region would generate a large gradient in $(Q, U)$. However, processes of that sort would also produce structure in the overall synchrotron emissivity, such that we would observe features in the image of Stokes $I$ that match those seen in $|\nabla \mathbf{P}|$. No such correspondence is observed, demonstrating that the regions of high polarization gradient are not intrinsic to the source of polarized emission but must be induced by Faraday rotation in magneto-ionized gas.

Because the amount of Faraday rotation is proportional to the line integral of $n_{e} B_{\|}$from the source to the observer (where $n_{e}$ is the density of free electrons and $B_{\|}$is the uniform component of the line-of-sight magnetic field), the filamentary structure seen in $|\nabla \mathbf{P}|$ must correspond to boundaries across which $n_{e}$ and/or $B_{\|}$show a sudden increase or decrease over a small spatial interval. Such discontinuities could be shock fronts or ionization fronts from discrete sources, as have been observed in polarization around the rims of supernova remnants, $\mathrm{H}$ II regions and planetary nebulae $\mathrm{e}^{11,18}$. We have examined this possibility by carefully comparing our image of $|\nabla \mathbf{P}|$ with images and gradient images of Stokes $I$ (tracing shock waves seen in synchrotron emission; ref. 11), 21-cm H I emission ${ }^{16}$ (tracing atomic hydrogen) and 656.3-nm $\mathrm{H} \alpha$ emission $^{19,20}$ (tracing ionized hydrogen) over the same field, but do not find any correspondences.

We conclude that the features seen in $|\nabla \mathbf{P}|$ are a generic component of diffuse, ionized gas in this direction in the sky. To test this hypothesis, we performed a series of three-dimensional isothermal simulations of magnetohydrodynamic turbulence in the ISM, each with different parameters for the sonic Mach number, defined as $M_{\mathrm{s}} \equiv\left\langle|\mathbf{v}| / \mathcal{c}_{\mathrm{s}}\right\rangle$, where $\mathbf{v}$ is the local velocity, $\boldsymbol{c}_{\mathrm{s}}$ is the sound speed and the averaging (indicated by angle brackets) is done over the whole simulation. For each simulation, we propagated a uniform source of polarized emission through the distribution of turbulent, magnetized gas. The resultant Faraday rotation produces a complicated distribution on the sky of Stokes $Q$ and $U$, from which we generated a map of the polarization gradient using equation (1). Images of $|\nabla \mathbf{P}|$ for representative simulations of the subsonic, transonic and supersonic regimes are shown in Fig. 3. Narrow, elongated filaments of high polarization gradient are apparent in each simulation in Fig. 3, although they differ in their morphology and degree of organization. In particular, the supersonic case (Fig. 3c) shows localized groupings of very high-gradient filaments, corresponding to ensembles of intersecting shocks $5^{5,21,22}$. By contrast, the subsonic (Fig. 3a) and transonic (Fig. 3b) cases show more-diffuse networks of filaments, representing the cusps and discontinuities characteristic of any turbulent velocity field ${ }^{6,21,23}$.

Visual comparison of the simulated distributions of $|\nabla \mathbf{P}|$ with real data (Fig. 2) suggests that the subsonic and transonic cases shown in Fig. 3a, b more closely resemble the observations than does the supersonic case. We can quantify this statement by calculating the thirdorder moment (skew, $\gamma$ ) and the fourth-order moment (kurtosis, $\beta$ ) of the probability distribution function of $|\nabla \mathbf{P}|$ for both observations and simulations: these quantities parameterize the degree of Gaussian asymmetry in the probability distribution function, and hence provide information on the amount of compression due to shocks in the data $^{6,24}$. 

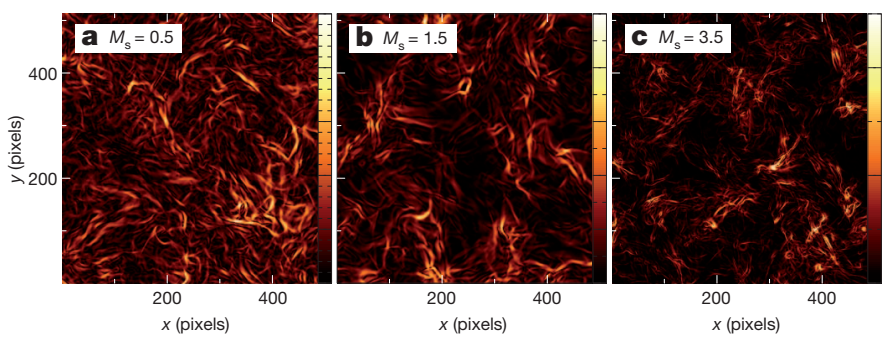

Figure $3|\nabla \mathrm{P}|$ derived from propagation of linear radio polarization through three different isothermal simulations of magnetized turbulence. Each simulation is a $512 \times 512 \times 512$-element periodic box with a linear dimension of $0.15 \mathrm{pc}$ for each pixel, evolved in time using an essentially nonoscillatory scheme ${ }^{24,30}$. Three such simulations are shown, each labelled with its corresponding value for $M_{\mathrm{s}}$ : subsonic $\left(M_{\mathrm{s}}<1 ; \mathbf{a}\right)$, transonic $\left(M_{\mathrm{s}} \approx 1 ; \mathbf{b}\right)$ and supersonic $\left(M_{\mathrm{s}}>1 ; \mathbf{c}\right)$. At the start of each simulation, the electron density had a uniform value $n_{e}=0.1 \mathrm{~cm}^{-3}$ and the magnetic field was oriented in the plane of the sky with a uniform amplitude of $B=0.3 \mu \mathrm{G}$ (subsonic), $1 \mu \mathrm{G}$ (transonic) or $2 \mu \mathrm{G}$ (supersonic), which corresponds to a constant Alfvénic Mach number of $M_{\mathrm{A}}=2$ in each case. Turbulence was driven solenoidally in Fourier space at large scales (small wavenumber) until the turbulent cascade had fully developed and a steady state between input energy and dissipation had been reached. In each case, we illuminated the simulation volume with a background radio source of uniform polarization at an emission frequency of $1.4 \mathrm{GHz}$, with $Q=100 \mathrm{mJy}$ per pixel and $U=0 \mathrm{mJy}$ per pixel at all positions. At each pixel, the line integral of $n_{e} B_{\|}$was computed, and the corresponding Faraday rotation was applied to the polarized signal, to calculate values of $Q$ and $U$. No effects due to finite angular resolution, depolarization or incomplete interferometric visibility coverage were included, so the observed polarized signal is $P=100 \mathrm{mJy}$ per pixel at all positions. We then calculated the gradient, $|\nabla \mathbf{P}|$, using equation (1). The scales showing $|\nabla \mathbf{P}|$ are shown on the right of the images, and range from 0 to $25 \mathrm{mJy}$ per (pixel) $)^{1.5}$ for a (interval, $1 \mathrm{mJy}$ per (pixel $^{1.5}$ ), 0 to $100 \mathrm{mJy}$ per (pixel) $)^{1.5}$ for $\mathbf{b}$ (interval, $10 \mathrm{mJy}$ per $(\text { pixel })^{1.5}$ ) and 0 to $500 \mathrm{mJy}$ per (pixel) $)^{1.5}$ for $\mathbf{c}$ (interval, $100 \mathrm{mJy}$ per (pixel) $)^{1.5}$ ).

In the simulations, we found that both the skew and the kurtosis of $|\nabla \mathbf{P}|$ increased monotonically with sonic Mach number. We used a genetic algorithm ${ }^{25}$ to determine that the threshold for strongly supersonic turbulence was $\gamma>1.1$ and $\beta>1.5$. We then computed the third- and fourth-order moments for the observed distribution of $|\nabla \mathbf{P}|$ shown in Fig. 2, and found that $\gamma=0.3$ and $\beta=0.9$.

This analysis of the moments of the polarization gradient therefore confirms quantitatively what we concluded above from visual inspection: the turbulent, ionized ISM in this direction in the sky is subsonic or transonic. The findings we obtained by imaging the polarization gradients produced by interstellar turbulence are supported by recent statistical studies of $\mathrm{H} \alpha$ emission measures and of 21-cm $\mathrm{H}$ I column densities over large volumes, which have similarly found that $M_{\mathrm{s}} \lesssim 2$ for warm gas throughout the ISM ${ }^{26,27}$.

In the simulations shown in Fig. 3, the sharp gradients in $(Q, U)$ occur as a result of localized high values of the gas density and magnetic field, resulting from vorticity or shock compression. However, the filamentary features seen in $|\nabla \mathbf{P}|$ may not be easily observable in other types of data: for example, if we adopt typical parameters for warm, ionized gas ${ }^{1,28}$ of $n_{e} \approx 0.3 \mathrm{~cm}^{-3}$ and $B_{\|} \approx 2 \mu \mathrm{G}$, even the compression associated with a strong adiabatic shock produces across-filament changes in emission measure and Faraday rotation measure of only $\sim 0.5 \mathrm{pc} \mathrm{cm}^{-6}$ and $\lesssim 5 \mathrm{rad} \mathrm{m}^{-2}$, respectively, assuming a spatial scale ${ }^{7,14}$ for these structures of $\sim 0.5 \mathrm{pc}$. This is below observable levels in $\mathrm{H} \alpha$ and other tracers of emission measure. The rotation measure gradient ${ }^{14}$ across these interfaces is potentially observable in spectropolarimetric radio data, but the addition of single-dish observations is required to recover the total power of the polarized signal. By contrast, even a small gradient in rotation measure can produce an arbitrarily large value of $|\nabla \mathbf{P}|$ (irrespective of whether single-dish measurements are present in the data), provided that there is a strong source of background polarized emission through which the discontinuities in Faraday rotation are viewed. Further investigation of the polarization gradient and its statistical properties will provide robust estimates of poorly constrained parameters of turbulent flows such as the sonic and Alfvénic Mach numbers, the characteristic magnetic field strength, the Reynolds number and the physical scale of energy injection.

Received 3 March; accepted 11 August 2011.

Published online 5 October 2011.

1. Ferrière, K. M. The interstellar environment of our galaxy. Rev. Mod. Phys. $\mathbf{7 3}$ 1031-1066 (2001).

2. de Avillez, M. A. \& Breitschwerdt, D. Global dynamical evolution of the ISM in star forming galaxies. I. High resolution 3D simulations: effect of the magnetic field. Astron. Astrophys. 436, 585-600 (2005).

3. Chepurnov, A. \& Lazarian, A. Extending the big power law in the sky with turbulence spectra from Wisconsin $\mathrm{H} \alpha$ Mapper data. Astrophys. J. 710, 853-858 (2010).

4. Armstrong, J. W., Rickett, B. J. \& Spangler, S. R. Electron density power spectrum in the local interstellar medium. Astrophys. J. 443, 209-221 (1995).

5. Kritsuk, A. G., Norman, M. L., Padoan, P. \& Wagner, R. The statistics of supersonic isothermal turbulence. Astrophys. J. 665, 416-431 (2007).

6. Kowal, G., Lazarian, A. \& Beresnyak, A. Density fluctuations in MHD turbulence: Spectra, intermittency, and topology. Astrophys. J. 658, 423-445 (2007).

7. Kissmann, R., Kleimann, J., Fichtner, H. \& Grauer, R. Local turbulence simulations for the multiphase ISM. Mon. Not. R. Astron. Soc. 391, 1577-1588 (2008).

8. Wieringa, M. H., de Bruyn, A. G., Jansen, D., Brouw, W. N. \& Katgert, P. Small scale polarization structure in the diffuse galactic emission at $325 \mathrm{MHz}$. Astron. Astrophys. 268, 215-229 (1993).

9. Gray, A. D., Landecker, T. L., Dewdney, P. E. \& Taylor, A. R. A large-scale, interstellar Faraday-rotation feature of unknown origin. Nature 393, 660-662 (1998).

10. Haverkorn, M., Katgert, P. \& de Bruyn, A. G. Structure in the local Galactic ISM on scales down to $1 \mathrm{pc}$, from multi-band radio polarization observations. Astron. Astrophys. 356, L13-L16 (2000).

11. Gaensler, B. M. et al. Radio polarization from the inner galaxy at arcminute resolution. Astrophys. J. 549, 959-978 (2001).

12. Uyanıker, B. \& Landecker, T. L. A highly ordered Faraday rotation structure in the interstellar medium. Astrophys. J. 575, 225-233 (2002).

13. Shukurov, A. \& Berkhuijsen, E. M. Faraday ghosts: depolarization canals in the galactic radio emission. Mon. Not. R. Astron. Soc. 342, 496-500 (2003).

14. Haverkorn, M. \& Heitsch, F. Canals beyond Mars: beam depolarization in radio continuum maps of the warm ISM. Astron. Astrophys. 421, 1011-1019 (2004)

15. Fletcher, A. \& Shukurov, A. Depolarization canals and interstellar turbulence. EAS Publ. Ser. 23, 109-128 (2007).

16. McClure-Griffiths, N. M. et al. The Southern Galactic Plane Survey: the test region. Astrophys. J. 551, 394-412 (2001).

17. Newton-McGee, K. J. Radio Polarimetry as a Probe of Interstellar Magnetism 83-87. Ph.D. thesis, Univ. Sydney (2009).

18. Ransom, R. R., Uyanıker, B., Kothes, R. \& Landecker, T. L. Probing the magnetized interstellar medium surrounding the planetary nebula Sh 2-216. Astrophys. J. 684, 1009-1017 (2008).

19. Gaustad, J. E., McCullough, P. R., Rosing, W. \& Van Buren, D. A robotic wideangle $\mathrm{H} \alpha$ survey of the southern sky. Publ. Astron. Soc. Pacif. 113, 1326-1348 (2001).

20. Parker, Q. A. et al. The AAO/UKST SuperCOSMOS Ha survey. Mon. Not. R. Astron. Soc. 362, 689-710 (2005).

21. Beresnyak, A., Lazarian, A. \& Cho, J. Density scaling and anisotropy in supersonic magnetohydrodynamic turbulence. Astrophys. J. 624, L93-L96 (2005).

22. Lemaster, M. N. \& Stone, J. M. Dissipation and heating in supersonic hydrodynamic and MHD turbulence. Astrophys. J. 691, 1092-1108 (2009).

23. Eyink, G. L. Besov spaces and the multifractal hypothesis. J. Stat. Phys. 78, 353-375 (1995).

24. Burkhart, B., Falceta-Gonçalves, D., Kowal, G. \& Lazarian, A. Density studies of MHD interstellar turbulence: statistical moments, correlations and bispectrum. Astrophys. J. 693, 250-266 (2009).

25. Whitley, D. A genetic algorithm tutorial. Stat. Comput. 4, 65-85 (1994).

26. Hill, A. S. et al. The turbulent warm ionized medium: emission measure distribution and MHD simulations. Astrophys. J. 686, 363-378 (2008).

27. Burkhart, B., Stanimirović, S., Lazarian, A. \& Kowal, G. Characterizing magnetohydrodynamic turbulence in the Small Magellanic Cloud. Astrophys. J. 708, 1204-1220 (2010).

28. Gaensler, B. M., Madsen, G. J., Chatterjee, S. \& Mao, S. A. The vertical structure of warm ionised gas in the Milky Way. Publ. Astron. Soc. Aust. 25, 184-200 (2008).

29. Haverkorn, M., Gaensler, B. M., McClure-Griffiths, N. M., Dickey, J. M. \& Green, A. J. The Southern Galactic Plane Survey: polarized radio continuum observations and analysis. Astrophys. J. 167 (suppl.), 230-238 (2006).

30. Cho, J. \& Lazarian, A. Compressible magnetohydrodynamic turbulence: mode coupling, scaling relations, anisotropy, viscosity-damped regime and astrophysical implications. Mon. Not. R. Astron. Soc. 345, 325-339 (2003).

Acknowledgements We thank S. Brown, A. Hill, R. Kissmann, A. MacFadyen, M.-M. Mac Low, E. Petroff, P. Slane and X. Sun for discussions. The Australia Telescope Compact Array is funded by the Commonwealth of Australia for operation as a National Facility managed by CSIRO. B.M.G. and T.R. acknowledge the support of the Australian Research Council through grants FF0561298, FL100100114 and FS100100033. B.B. acknowledges support from the National Science Foundation Graduate Research Fellowship and the NASA Wisconsin Space Grant Institution. A.L. acknowledges the support of the National Science Foundation through grant ASTO808118 and of the 
Center for Magnetic Self-Organization in Astrophysical and Laboratory Plasmas. We thank the staff of the Australia Telescope National Facility, especially M. Calabretta, R. Haynes, D. McConnell, J. Reynolds, R. Sault, R. Wark and M. Wieringa, for their support of the Southern Galactic Plane Survey.

Author Contributions J.M.D., N.M.Mc.-G., B.M.G. and A.J.G. carried out the original observations. B.M.G., N.M.Mc.-G. and T.R. produced the polarization images from the raw data. B.M.G., M.H., K.J.N.-Mc., R.D.E and N.M.Mc.-G. worked together to develop the gradient technique, and B.M.G. then applied the gradient technique to the images. B.B. and A.L. performed the simulations and the statistical analysis. B.M.G. led the writing of the paper and the interpretation of results. All authors discussed the results and commented on the manuscript.

Author Information Reprints and permissions information is available at www.nature.com/reprints. The authors declare no competing financial interests. Readers are welcome to comment on the online version of this article at www.nature.com/nature. Correspondence and requests for materials should be addressed to B.M.G. (bryan.gaensler@sydney.edu.au). 